\title{
Spectacles des corps et premier cinéma
}

spectacles of the body and early cinema

\section{Patrick Désile}

\section{(2) OpenEdition}

\section{Journals}

Édition électronique

URL : https://journals.openedition.org/aes/4339

DOI : 10.4000/aes.4339

ISSN : 2258-093X

Éditeur

Laboratoire LISAA

Référence électronique

Patrick Désile, "Spectacles des corps et premier cinéma », Arts et Savoirs [En ligne], 16 | 2021, mis en ligne le 17 décembre 2021, consulté le 19 décembre 2021. URL : http://journals.openedition.org/aes/ 4339 ; DOI : https://doi.org/10.4000/aes.4339

Ce document a été généré automatiquement le 19 décembre 2021.

Centre de recherche LISAA (Littératures SAvoirs et Arts) 


\title{
Spectacles des corps et premier cinéma
}

\author{
Spectacles of the body and early cinema
}

Patrick Désile

1 Que le premier cinéma soit un spectacle des corps, on en convient volontiers. Ce sont des corps, en effet, marchant, courant, sautant, tombant, qu'il montra d'abord. Il ne montra pas cela seulement, sans doute, il montra aussi de la fumée, des vagues, des feuilles... le monde, enfin, palpitant, et c'est ce qui faisait de lui autre chose qu'un chronophotographe. Mais enfin l'objet principal de ces premières bandes, c'était tout de même bien cela, ces corps animés, ces ouvrières sortant de l'usine, ces soldats à l'exercice, ces forgerons au travail, ces baigneurs, ces acrobates, ces danseuses...

2 Et que le cinéma, spectacle des corps, ait quelques relations avec d'autres spectacles des corps, contemporains de son émergence ou de peu antérieurs, nul n'en doute, cela se dit très bien. Cirque, café-concert, spectacles forains, ou bien figures de cire, exposition des cadavres à la morgue, exhibitions de la Salpêtrière... Longtemps, les plus apparentes de ces relations donnèrent lieu à des propos convenus, insuffisamment informés, et les poncifs émaillent encore bien des discours. Mais l'on a commencé à poser, sur les spectacles de curiosité, par exemple - beaucoup des spectacles dont nous parlerons ici relèvent de cette catégorie juridique -, un regard moins désinvolte. Et ces dernières années, un certain nombre de travaux érudits, pertinents, ont mis en évidence des liens inaperçus entre tels spectacles des corps et le premier cinéma ${ }^{1}$.

Il semble pourtant qu'il faille revenir sur ces relations, pour plusieurs raisons.

4 D'abord, et malgré d'heureuses remises en question des présupposés téléologiques qui inspiraient souvent les discours sur le "pré-cinéma " - et cette expression même, encore volontiers employée, en témoigne -, quelque chose semble parfois subsister de ces anciennes approches: le cinéma "prolongerait ", "continuerait» tels spectacles des corps, tels autres l'auraient « préparé »... C'est qu'il n'est pas aisé, sans doute, pour homo cinematographicus, de se déprendre tout à fait d'un discours qui adhère au cinéma, qui s'est constitué en même temps que lui, pour lui. Au demeurant, il y eut bien, en 
effet, des transferts, des emprunts, la perpétuation d'une culture par d'autres moyens, et ce schéma continuiste n'est pas, bien entendu, absolument invalide, tant s'en faut ; on doit seulement se garder d'en faire un principe quasi exclusif d'intelligibilité.

Il se peut d'ailleurs que cette sorte d'illusion de continuité soit favorisée par le fait que les recherches qui tentent de faire apparaître de telles relations ne s'attachent qu'à un nombre limité de spectacles. Cela non plus n'est pas illégitime, mais il se trouve que les spectacles des corps ont été nombreux pendant la période de l'émergence du cinéma ; or, beaucoup d'entre eux sont rarement pris en considération ou ne le sont jamais.

Nous proposons donc d'envisager un ensemble beaucoup plus large de ces spectacles des corps - sans nous soucier des liens qu'on pourrait leur supposer avec le cinéma émergent -, d'esquisser, à l'intérieur de cet ensemble, des lignes d'évolution, et de nous demander comment le cinématographe s'y insère. Il va sans dire que dans le cadre limité de cet article, nous ne pourrons donner qu'un aperçu très sommaire de cette matière immense et ébaucher seulement quelques pistes d'intelligibilité.

7 Entre 1880 et 1914, donc, les spectacles des corps semblent se multiplier. À Paris, en tout cas, et c'est Paris qui nous retiendra surtout (à l'instar de Londres, Vienne ou NewYork, le Paris de 1900 est en effet «un extraordinaire laboratoire de la modernité culturelle $»^{2}$ ). Mais il faut préciser ce que nous entendons ici par "spectacles des corps ». Par "spectacle ", d'abord: on ne peut se limiter à l'acception restreinte de ce mot dans l'expression " arts du spectacle », car d'autres spectacles existent, clairement désignés comme tels, qui ne relèvent pas de cette catégorie; on ne saurait pourtant l'étendre à l'infini et tenir ici pour spectacle « tout ce qui attire le regard, l'attention, arrête la vue $»^{3}$ (bien que ce soit la première définition que Littré donne de ce mot). Nous prendrons une voie médiane en tenant pour « spectacle » tout ce qui est fait pour être vu par un public (même restreint) expressément convoqué ou rassemblé.

nous intéresserons seulement, donc, aux "spectacles des corps ». Qu' est-ce à dire? Nous gardant, là encore, d'une définition trop restrictive de "corps", nous considérerons comme "spectacles des corps " ceux dans lesquels le corps - corps vivant ou corps sans vie, corps réel ou simulacre, corps charnel ou désincarné, corps humain ou corps animal - est le principal objet de l'attention. Il faut d'ailleurs noter même si nous ne pourrons guère développer ce point - que ce n'est pas le seul corps exhibé qui devrait être pris en compte, mais bien aussi celui du spectateur; corps regardé et corps regardant ne peuvent pas être dissociés ni même, parfois, distingués.

9 Ces prolégomènes posés, il nous faut dresser un inventaire de ces spectacles des corps. Inventaire nécessairement succinct, d'ailleurs non-exhaustif ${ }^{4}$, on l'a dit.

Il convient, sans doute, d'évoquer d'abord le spectacle des corps par excellence, la danse ${ }^{5}$. Mais - et ce sera le cas, à un degré à peine moindre, pour tous les spectacles que nous considérerons - il n'est pas envisageable de faire plus ici qu'effleurer ces mondes de la danse, ces déclinaisons infinies des corps en mouvement. Corps harmonieux (alors presque exclusivement féminins) des ballets de l'Opéra (qui subsistent avec succès, bien que la fin de siècle les voie en déclin), corps capiteux du french cancan, corps étranges et troublants des danses exotiques (ainsi les danses javanaises de l'Exposition universelle de 1889, accompagnées par un gamelan, auxquelles assiste Debussy...). Corps évanescent de Loïe Fuller, aussi, dissous dans les voiles et les lumières, que Paul Adam voit se « décorporifier $»^{6}$ et qui suscite tant d'admirations et d'imitations dans les années $1890 . .$. 
11 On doit mentionner encore les nombreuses danses de salons - et l'on sait l'importance que revêt alors le bal comme espace de sociabilité et de visibilité -, danses anciennes ou régionales, qui connaissent une nouvelle vogue, ou danses nouvelles, notamment celles, qui, venues d'Amérique, suscitent un véritable engouement (le boston, la mattchiche, le cake-walk dans les premières années du $\mathrm{xx}^{\mathrm{e}}$ siècle, le tango un peu plus tard...).

Puis il y a les corps remarquables, souvent agités, cocasses, des artistes des cafésconcerts $^{7}$ - Paulus le "gambillard", Bourgès ou Clovis, les «poivrots", le gros Mansuelle, la corpulente Jeanne Bloch, « la plus comique du monde entier ${ }^{8}$, Dorville, qui imite le phoque, Sinoël, « original, pittoresque, truculent, épileptique ${ }^{9}$, Polaire, qui « se trémousse, frétille, balle des hanches, des reins et du nombril, mime toutes les secousses, se tord, se cambre, se cabre $[. ..] »^{10}$.

13 La prestidigitation, sur diverses scènes, malmène aussi volontiers les corps : disparitions, décapitations, démembrements ${ }^{11} \ldots$

14 La pantomime ${ }^{12}$, art corporel s'il en est, qu'on avait dite en déclin, connaît un renouveau à la fin du siècle, qu'illustrent la création du Cercle funambulesque en 1888, les pantomimes écrites par Huysmans, Catulle Mendès, Jean Richepin, le succès de Georges Wague et de Félicia Mallet... De cette attention nouvelle à la gestuelle témoigne la publication du Geste ${ }^{13}$, de Charles Hacks, de Notation des gestes ${ }^{14}$ de Georges Polti, de L'Art mimique ${ }^{15}$ de Charles Aubert...

15 C'est une pantomime aussi, mais d'un genre assez différent, que le Coucher d'Yvette qu'interprète Blanche Cavelli au concert Lisbonne en 1894: une femme se déshabille, simplement, puis se couche. Le succès est tel que, dans les dix-huit mois qui suivent, on donne à Paris plus de vingt-cinq pantomimes dont l'essentiel est une scène de déshabillage féminin... Le «nu au théâtre » (c'est le titre d'une rubrique dans plusieurs journaux ${ }^{16}$ ) prospère; à partir de l'effacement de la censure théâtrale et particulièrement pendant toute l'année 1908, des spectacles aux titres aguichants (et souvent trompeurs) fleurissent, variations obsessionnelles sur le thème du nu: Imposons la revue nue ${ }^{17}$, Paris tout $n u^{18}$, Nue cocotte $e^{19}$, As-tu vu mon $n u^{20}, J^{\prime} v e u x d u ~ n u, ~ n a ~ !^{21}, \grave{A}$ nu les femmes !22 ... En 1909, paraît Le $\mathrm{Nu}$ au théâtre depuis l'Antiquité jusqu'à nos jours, ouvrage abondamment illustré, écrit par deux médecins, qui veut être « un plaidoyer pour le nu, un réquisitoire contre l'obscène $»^{23}$ et s'ouvre ainsi : «En voulez-vous du $\mathrm{nu}$ ? On en met partout $»^{24}$.

16 Ces spectacles sont plus grivois que vraiment impudiques : le maillot couleur chair, la chemise, le costume font en général écran. Mais en 1893, au bal des Quat'z'arts, donné au Moulin-Rouge, le modèle Sarah Brown s'était présentée buste nu et jambes nues sous une résille à larges mailles et sans maillot (tenue dans laquelle elle avait posé pour la Cléopâtre de Rochegrosse). Un procès s'était conclu par un jugement clément. Au début $\mathrm{du} \mathrm{xx}^{\mathrm{e}}$ siècle, à l'occasion d'un autre procès, intenté à quatre établissements (l'Olympia, les Folies Pigalle, les Folies royales et le Little Palace) qui ont présenté des scènes de nu, la jurisprudence établit enfin à quelles conditions le nu sur scène est acceptable :

Le fait de représenter ou faire représenter au théâtre des scènes dans lesquelles figurent des femmes nues ne constitue pas le délit d'outrage public à la pudeur, lorsqu'il résulte des diverses précautions prises, des jeux de lumière combinés, de la disposition des gazes, de l'éloignement des actrices par rapport au public, de leurs poses purement plastiques, immobiles et dégagées de toute intention lascive, que les directeurs et artistes n'ont entendu procurer au public qu'une impression d'art et de beauté plastique, exclusive de tout scandale susceptible de blesser la pudeur des spectateurs. ${ }^{25}$ 

peuplent les expositions ethnographiques. Indiens Galibis, Kalmouks, Achantis, Hottentots, Somalis, Caraibes se succèdent par exemple au Jardin d'acclimatation au cours des décennies 1880 et 1890. À l'Exposition universelle de 1900, « ...les villes et les villages algériens, tunisiens, sénégalais, cochinchinois, canaques, etc., sont habités par des gens venus du pays même $»^{32}$. 

sont exposés, à des fins de reconnaissance ${ }^{36}$. Les guides de Paris mentionnent l'établissement, ouvert à tous, tous les jours de l'année. L'affluence est parfois considérable: "La foule, qui s'écrase à certains jours devant les vitrines de la salle d'exposition, n'y vient chercher que des émotions violentes; ce n'est pour elle qu'un spectacle à sensation, permanent et gratuit. $\|^{37} \mathrm{Il}$ arrive que les corps soient apprêtés et mis en scène: en 1886, une petite fille, découverte rue du Vert-Bois, est présentée assise (et non allongée), sur une chaise garnie d'une étoffe rouge. $\mathrm{Au}$ cours de la nuit qui précède l'exécution, une foule où « les hauts-de-forme cottoient [sic] les casquettes plates $»^{38}$ se presse vers «le théâtre de la place de la Roquette $»^{39}$; dans les cafés restés ouverts, on boit, on plaisante, on rit. Enfin le couperet tombe, et la foule se disperse pendant que l'on emporte le corps et la tête.

D'autres corps sans vie font l'objet d'une exhibition, pour un public restreint, il est vrai : ce sont les cadavres destinés aux leçons d'anatomie, et l'on pense moins ici au public spécialisé de l'école de médecine qu'à celui plus large de l'école des beaux-arts : pendant tout le XIX ${ }^{e}$ siècle, la dissection est censée concourir à la formation de l'artiste.

31 Il est encore d'autres spectacles de corps sans vie : apparitions, fantômes... Le public est peu nombreux, bien sûr. En août 1905, à la villa Carmen, à Alger, sous les yeux d'une dizaine de personnes (parmi lesquelles le général Noël, polytechnicien, propriétaire de la villa, et Charles Richet, futur prix Nobel de médecine), un spectre qui prend parfois la forme d'un homme vêtu de blanc et se fait appeler Ben Boa, se matérialise à plusieurs reprises. En 1906, à la bibliothèque de l'Institut général psychologique, alors que la medium Eusapia Palladino est liée sur une chaise longue, derrière les tentures de son 
cabinet noir, un aréopage de savants voit « apparaître pendant un instant à la fente du rideau comme une tête obscure et un buste d'homme recouverts de linges blancs ${ }^{40} \ldots$ On peut bien entendu tenir ces apparitions pour de simples effigies, et les illusionnistes se font fort de démontrer, pour un public plus large, qu'elles ne sont rien d'autre, en effet.

Mais d'autres effigies du corps humain, celles-là incontestablement matérielles, peuvent encore être évoquées, celles, en particulier, qui se donnent pour des imitations parfaites, comme les figures de cire. Le matériau même, utilisé de longue date pour des moulages, emporte l'idée de substitut. Le musée Grévin, inauguré en 1882, a pour principes « la reproduction fidèle de la nature et le respect de la vérité jusque dans les moindres détails $»^{41}:$ "chaque personnage est représenté dans son attitude familière, revêtu de ses propres vêtements ; les mains moulées sur nature $»^{42}$. Des musées de cires historiques et d'actualités figurent aussi dans les foires, tel le Grand Musée Buiron, avec ses "groupes à sensation" (Napoléon $1^{\text {er }}$ entouré de son état-major, Louis XIV et son siècle...) et ses « célébrités du jour " (Léon Gambetta, Garibaldi, Louise Michel.... ${ }^{43}$.

D'autres cabinets de figures de cire montrent le corps intime, secret, charnel et anonyme, souvent souffrant : les musées anatomiques. On y voit la Vénus qui respire ou l'homme mort de faim et, dans les salons réservés (aux hommes), des hermaphrodites et des syphilitiques. Le plus célèbre de ces musées est celui du docteur Spitzner, qui, installé d'abord place du Château d'eau, est devenu itinérant dans les années 1880, mais ces établissements sont nombreux dans les fêtes foraines de la fin du siècle.

D'autres effigies, qui ne se veulent pas, sans doute, d'un réalisme aussi scrupuleux, et qui obtiennent un succès plus local, doivent pourtant retenir l'attention: plusieurs spectacles, dans les dernières décennies du siècle, présentent des marionnettes perfectionnées, censées restituer à la perfection les mouvements du corps vivant. Les "maquettes animées" de Georges Bertrand, en 1890, "se meuvent et gesticulent comme des personnes naturelles » ${ }^{44}$, le "théatre mécanique " (mais il s'agit bien de marionnettes) de John Hewelt, dans les années 1890, « a l'animation, la vie d'une vérité parfaite $»^{45}$, les Bonshommes Guillaume de l'Exposition universelle de 1900 ont «l'indépendance, le mouvement, la souplesse et l'apparence de la vie » ${ }^{46}$.

Mentionnons encore des spectacles qui sont à la fois des spectacles de corps vivants et des spectacles d'effigies, puisqu'ils inversent le rapport des uns et des autres : dans les tableaux vivants, les modèles imitent les représentations statuaires ou picturales ${ }^{47}$. Au Musée vivant de Melchior Bonnefois, dans les années 1880, on admire des représentations du Serment des Horaces, du Radeau de la Méduse, des tableaux des salons des années 1880 ou des groupes sculptés, L'Enlèvement de Polyxène de Pio Fedi, Quand même! d'Antonin Mercié48...

On ne saurait enfin ignorer les spectacles des corps animaux. Au cirque, les bêtes sauvages dominées, dociles, presque humanisées du début du XIX ${ }^{e}$ siècle ont fait place à des bêtes féroces, et domptées, certes, exécutant des tours, mais farouchement animales. Dans les suppléments des journaux populaires s'étalent les récits et les images de dompteurs (et parfois du public) attaqués par quelque fauve retourné à ses instincts.

37 Les combats d'animaux sont censés avoir disparu vers le milieu du XIX siècle (Colette se souvenait pourtant, aux dires d'Henry Thétard, d'avoir assisté, enfant, dans les années 1880 , lors du passage d'un cirque misérable, à «la bataille que des dogues décharnés livraient à un pauvre âne affublé d'un masque de cuir $»^{49}$ ). Mais alors paraissent les 
courses de taureaux. Les corridas sont assez fréquentes dans le Midi dès les années 1850 , et bientôt on peut en voir dans les villes du Nord, surtout à partir des années 1880. À Roubaix, en 1899, on fait s'affronter un lion et un taureau. À Paris, en 1889, une véritable arène a été construite.

Voici donc brièvement esquissé ce tableau des spectacles des corps entre 1880 et 1914. Il faut remarquer que beaucoup d'entre eux sont associés à des lieux, spécifiques ou non: le cirque, le café-concert, la fête foraine, pour les spectacles des corps extraordinaires, l'amphithéâtre, la morgue, la place de la Roquette puis le boulevard Arago pour les spectacles des corps sans vie, l'hôpital, le salon, la salle de bal, la place publique, la rue, pour les spectacles des corps ordinaires... La ville est ainsi constellée de spectacles des corps.

Quelques-uns sont anciens, mais bien peu, sous la forme qu'ils revêtent à la fin du XIX siècle, sont antérieurs à la fin du siècle précédent. Et beaucoup sont apparus dans la seconde moitié du siècle, ont souvent connu alors une sorte d'âge d'or, ou sont même spécifiques de l'époque.

C'est le cas pour le café-concert, qui émerge sous le Second Empire mais connaît un développement considérable sous la Troisième République. En 1911, comme on lui reproche de se montrer excessivement tolérant à l'égard des cafés-concerts, le préfet de police Lépine répond: «[...] nous avons à Paris, tous les soirs, 400 à 500, peut-être 600 , peut-être 700 salles ouvertes au public $[. ..] »^{50}$.

41 L'histoire du cirque s'inscrit davantage dans la longue durée, mais les cirques stables sont alors plus nombreux qu'ils ne l'ont jamais été: cirque d'Hiver, cirque d'été (jusqu'en 1900), cirque Fernando, qui devient Medrano en 1897, Nouveau Cirque à partir de 1886, cirque Métropole à partir de 1906, auxquels on peut ajouter le cirque amateur (et très mondain) Molier, et les hippodromes : hippodrome du Pont de l'Alma de 1877 à 1892, cirque-hippodrome du Champ-de-Mars de 1892 à 1900, hippodrome de la place Clichy en 1900.

Les spectacles de danse ne sont évidemment pas non plus propres à ces années-là, mais on a déjà noté le poids de la danse dans la vie sociale, l'extrême engouement pour des danses à la mode, la fascination qu'exercent les danses orientales ou la danse serpentine de la Loïe Fuller. La pantomime connaît un renouveau éclatant, suscite l'intérêt des écrivains et des intellectuels. Les pantomimes grivoises, les spectacles de nu (nudité relative, certes) se multiplient. La fête foraine connaît aussi une sorte d'âge d'or, les exhibitions de phénomènes, les musées d'anatomie, les théâtres de tableaux vivants attirent un public nombreux.

Dans l'espace public, les démonstrations de gymnastique ou les défilés militaires sont fréquents. L'intérêt pour les manifestations sportives conduit à l'organisation de compétitions : championnat de France de football en 1894, Jeux olympiques de Paris en 1900, Tour de France en 1903... On connait d'ailleurs l'engouement pour la « reinebicyclette » dès la fin du xix ${ }^{e}$ siècle.

Cette efflorescence semble traduire un désir effréné de voir des corps, de les voir dans tous leurs états, sous toutes les coutures. Cet ensemble fait en quelque sorte système, et si l'on dresse une typologie des corps exhibés, on peut mettre, en regard du caractère principal de chacun d'eux, son contraire, comme s'il s'était agi de parcourir tout le spectre des possibilités de montrer et de voir des corps. Dans ce vaste magasin d'exhibition des corps, il y a des corps humains et des corps animaux; des corps vêtus 
ou travestis et des corps nus; des corps vivants et des corps morts (sur l'échafaud, à la morgue, sur les tables de dissection) ; des corps féminins et des corps masculins (et bien que leur répartition soit peut-être plus complexe qu'il n'y paraît d'abord, on peut noter que les premiers sont plutôt censés être du côté de la passivité, les seconds de l'activité); il y a des corps charnels (corps plus ou moins dénudés d'actrices, de modèles, de prostituées, corps d'athlètes presque nus) et des corps évanescents (corps de Loïe Fuller et de ses imitatrices, corps des fantômes) ; des corps parfaits (danseuses, gymnastes, acrobates) et des corps difformes (phénomènes) ; des corps opaques et des corps translucides (spectres, corps soumis aux rayons $\mathrm{X}$ ) ; des corps intègres et des corps morcelés (fictivement dans les spectacles de magie, figurativement dans les musées anatomiques, réellement sur la table de dissection, sur l'échafaud, parfois à la morgue); des corps étiques et des corps obèses, des corps nains et des corps géants (dans les entre-sort, notamment); des corps sains (gymnastes et sportifs) et des corps malades (présentés lors des leçons cliniques ou, en effigie, dans les musées anatomiques); des corps autochtones et des corps exotiques; des corps autonomes et des corps possédés ; des corps domptés et des corps sauvages; des corps mobiles et des corps immobiles ; des corps souples et des corps ankylosés, paralysés...

Et l'on peut ajouter qu'à ces relations de réciprocité et de complémentarité s'en ajoutent d'autres, peut-être plus occultes, en particulier entre les spectacles qui regardent du côté de la médecine et ceux qui sont censés relever du pur divertissement. Les phénomènes des entre-sort peuvent évidemment être objets d'investigations et de présentations scientifiques autant que de monstrations récréatives. Mais les relations sont parfois plus complexes : Auguste Tuaillon et Delphin Sirvaux, qui mesurent l'un et l'autre environ un mètre, sont examinés et présentés à la société d'anthropologie par Léonce Manouvrier ${ }^{51}$; ils sont des " phénomènes ", si l'on veut, mais ils sont surtout des artistes de café-concert et de cabaret reconnus et des acteurs accomplis; la carrière cinématographique de Delphin est d'ailleurs remarquable (Le Nain, de Louis Feuillade, Zéro de conduite de Jean Vigo...).

Le fantaisiste Brunin est seulement un peu moins maigre que l'homme-squelette, Little Tich pourrait presque figurer dans un entre-sort comme nain polydactyle ${ }^{52}$ ou tel contorsionniste comme homme-caoutchouc, et de là dans quelque amphithéâtre... Les prestations de certains artistes du café-concert, notamment ceux qui relèvent du genre " épileptique », comme Polaire, ont d'ailleurs de flagrantes analogies avec les poses des hystériques de la Salpêtrière ${ }^{53}$. Et c'est à la Salpêtrière que Jeanne Louise Beaudon, patiente de Charcot, se découvre danseuse avant de triompher, sous le nom de Jane Avril, sur les scènes du Moulin Rouge et du Divan japonais.

Il est des correspondances plus sombres. En septembre 1880, alors que la foule se presse pour assister à l'exécution de Louis Menesclou, un cabinet de figures de cires, le musée du Progrès, s'assure un grand succès à la fête des Loges en affichant à sa porte : «Venez voir l'horrible crime de la rue de Grenelle, Menesclou découpant sa victime âgée de 4 ans ${ }^{54}$. L'école pratique de la faculté de médecine récupère alors les corps des décapités immédiatement après l'exécution : celui de Menesclou est soumis à des expériences injection du sang d'un chien, décharges électriques...- et démembré. Le cerveau est prélevé et moulé (son examen révèle qu'on a "guillotiné un fou, un malade, une sorte de bête fauve n'ayant de l'homme que l'apparence extérieure ${ }^{55}$ ), puis le moulage va rejoindre les collections du laboratoire d'anthropologie. 
Mais il y a, à vrai dire, une infinité de correspondances, de jeux de miroir, de circulations d'un espace à l'autre. Et entre le cinéma, qui émerge alors, et ces spectacles, on peut aussi établir de telles relations. Non seulement il emprunte des contenus, des figures, prolonge une culture ordinaire par ses moyens propres - et l'on verra bien sûr d'emblée au cinéma les danseuses et les acrobates, les couchers d'Yvette et les défilés militaires, et des corps morcelés, escamotés, hypnotisés...-, mais les corps qu'on y voit s'expriment (du moins dans les films narratifs) par le geste, comme les mimes de la pantomime théâtrale, ils ont la même ressemblance mimétique avec leurs modèles que les figures de cire, ils restituent la vérité des mouvements des corps comme les marionnettes fin de siècle (et mieux qu'elles), ils peuvent se figer comme les tableaux vivants et, s'ils donnent à voir la chair même, ces corps-là sont aussi par essence translucides, spectraux. Le cinéma a d'ailleurs - c'est un poncif d'emblée quelque accointance avec l'au-delà, lui qui sauvegarde la vivante apparence des corps au-delà même de la mort.

On pourrait décrire plus précisément ces relations (et d'autres), analyser les spécificités, les singularités, les complexités. Mais il importe de se demander d'abord si le cinéma intervient dans cet ensemble en quelque sorte accidentellement, prolongeant tel spectacle, évinçant tel autre, ou si son émergence s'inscrit dans un schéma plus général. Nous avons très allusivement indiqué quelques lignes d'évolution, mais il nous faut y revenir, au moins pour quelques-uns de ces spectacles.

50 Un certain nombre d'entre eux, en effet, ne survivent guère au $\mathrm{XIX}^{\mathrm{e}}$ siècle ou connaissent, du moins, à l'orée $d u x^{e}$ siècle, de profondes mises en cause. C'est en particulier le cas des spectacles des corps sans vie. Le spectacle des exécutions capitales, bien qu'il attire les foules (et parce qu'il les attire, sans doute) connaît une occultation progressive. Dès 1851, pour épargner le transfert au condamné, la guillotine se dresse tout près de la prison, place de la Roquette ; l'échafaud est supprimé en 1872 ; on tient fermement la foule à distance; les exécutions ont lieu au petit jour. Le caractère public de l'exécution, malgré ces restrictions qui la rendent difficilement visible, est cependant maintenu, puisqu'elle est censée avoir une valeur d'exemplarité.

51 Le nombre des exécutions va diminuant, cependant, tout au long du siècle. Dans les années 1880 et 1890, il n'y a guère, à Paris, qu'une ou deux exécutions par an, rarement trois. Mais il n'y en a aucune entre 1895 et 1897, aucune entre 1900 et 1908 : Félix Faure et Émile Loubet accordent volontiers la grâce, Armand Fallières l'accorde systématiquement. Et la peine capitale est tout près de disparaître du Code pénal : en 1908, un projet de loi d'abolition est soumis aux députés. Il est cependant rejeté : en 1907, le viol et l'assassinat d'une petite fille par Soleilland et la grâce qui lui a été accordée ont soulevé l'indignation. Une campagne de presse en faveur du maintien de la peine de mort et surtout de son application porte ses fruits : l'abolition est écartée et Fallières se résout à refuser des grâces. En 1909, on exécute devant la prison de la Santé le parricide Duchemin. Mais le spectacle de la décapitation avait bien failli disparaître et il disparait, en tout cas, pour un temps, des écrans. Clemenceau avait en effet interdit avec vigueur, lors de la reprise des exécutions, à Béthune, le 11 janvier 1909, la diffusion de prises de vues qui auraient pu y être faites, et, à cette occasion, la diffusion de toute image de décapitation, même fictive. Cette interdiction marque la naissance de la censure cinématographique en France.

52 L'exposition à la morgue connaît un sort plus radical. Dès les années 1880, et surtout après la publication, en 1887, de l'ouvrage du juge d'instruction Adolphe Guillot Paris 
qui souffre ${ }^{56}$, des voix s'élèvent pour que l'exposition publique des cadavres, considérée comme immorale, obscène, inutile, cesse. Malgré la foule qui afflue toujours, malgré Le Petit Journal qui réclame son maintien, elle est définitivement interdite le 15 mars 1907.

D'autres spectacles alors populaires, comme certains spectacles des fêtes foraines, connaissent un sort comparable. Le préfet de police interdit certains manèges réputés inconvenants, comme les « tonneaux de l'amour » et certains spectacles :

Les exhibitions de phénomènes vivants, les spectacles présentant un caractère obscène ou répugnant, les exhibitions de femmes, sous quelque forme que ce soit et généralement tous spectacles dits Entre-Sort, sont formellement interdits. ${ }^{57}$

Aux musées anatomiques, il impose de sévères restrictions :

Des pièces anatomiques seront seules exposées ;

Ces pièces n'offriront aucun caractère répugnant ou de nature à provoquer de la part des visiteurs des réflexions obscènes ou contraires aux bonnes mœurs ;

Il n'y aura pas de musée secret accessible à une catégorie spéciale de visiteurs ;

Aucun sujet lascif ne sera représenté..$^{58}$

Ces mesures d'interdiction sont inégalement respectées, mais elles ne sont pas sans portée. Il se peut d'ailleurs qu'elles confortent une évolution de l'opinion : alors que, dans les années 1880 , on ne compte pas moins de six à huit musées d'anatomie dans les grandes fêtes foraines, il n'y en a plus que trois ou quatre de 1890 à 1911, deux seulement en 1912 et $1913^{59}$. Ils seront définitivement interdits en 1921.

Nous avons dit que la dissection avait fait partie du cursus de l'école des Beaux-Arts pendant tout le XIXe siècle. Le professeur d'anatomie nommé en 1903, Paul Richer, élève de Charcot et l'un des principaux artisans de l'Iconographie de la Salpêtrière, néglige la dissection et privilégie la photographie et la chronophotographie. Son adjoint, Georges Chicotot, s'intéresse aux rayons $\mathrm{X}^{60}$. La dissection disparaît de l'enseignement des beaux-arts, emportée par les nouvelles imageries.

D'autres spectacles, associés, sans doute, à l'angoisse fin de siècle devant le corps, semblent tomber en disgrâce: théâtre de l'hystérie, petit théâtre du spiritisme scientifique... Le café-concert, même, en ce qu'il était souvent un spectacle du corps banal tendanciellement détraqué ou monstrueux, cède la place. Les cabinets secrets, les spectacles plus ou moins obscènes, désertent peu à peu les foires.

D'autres spectacles, enfin, émergent ou s'épanouissent : spectacles mettant en scène la nudité (mais relative, esthétisée), spectacles de corps d'exception (de gymnastes, d'athlètes, d'acrobates en partie dénudés, ou laissant au moins deviner leurs formes). Faut-il donc voir, dans ces évolutions, un effacement des spectacles de la mort au profit de spectacles de la nudité ? En 1955, l'anthropologue Geoffrey Gorer avait soutenu, dans un article demeuré célèbre ${ }^{61}$, qu'au $x^{e}{ }^{e}$ siècle, par une sorte d'inversion de la situation antérieure, la sexualité avait cessé d'être taboue, alors que la mort l'était devenue. Et il parlait, à juste titre, sans doute, de l'émergence d'une "pornographie de la mort». Mais l'évolution ne se résume pas selon nous à ce renversement des pornographies.

Le spectacle de la mort ne devient pas, en effet, absolument tabou: le cinéma, en particulier, donne très tôt à voir des scènes macabres : Histoire d'un crime, de Ferdinand Zecca, en 1901, inspiré de tableaux du musée Grévin, montre un assassinat, la morgue et l'exécution du coupable, et plus tard, c'est la multiplication de scènes de crimes qui justifie la campagne de censure de 1912. Les spectacles de la nudité, d'autre part, ne sont que tolérés, et à la condition d'une esthétisation, d'une mise à distance. 
60 Ce qui s'efface, en réalité, ce n'est pas tant le spectacle de la mort que celui des corps exagérément présents, crus, vulgaires, précaires, souffrants, anormaux, imparfaits, et périssables, mortels enfin: morgue, décapitation, dissection, viscères, corps tordus et grimaçants. Si des spectacles de corps qu'on pourrait dire naturels subsistent, ce sont précisément des spectacles d'animaux, et sauvages, d'ailleurs très encadrés. Et ce qui se fait jour, c'est moins le spectacle de corps érotisés que celui de corps non seulement apprêtés, façonnés, mais mis à distance, mis en scène, et comme fictionnalisés. Or, ce nouveau régime de visibilité, le cinéma en est non seulement le parfait instrument, mais il en est, d'une certaine manière, la matérialisation. Le cinéma montre tout, sans doute, mais de façon médiate : il fictionnalise les corps dont il capture l'apparence et les assujettit au temps qui est le sien, réversible et reproductible, et les insère d'emblée dans un récit.

61 Au demeurant, il ne montre pas tout à fait tout, dans un premier temps. Il est révélateur en effet que, comme nous l'avons dit, ce soit l'interdiction de montrer des décapitations réelles (et cette coupure du corps en deux avait hanté tout le XIX ${ }^{e}$ siècle) qui inaugure et fonde la censure cinématographique en France. On peut aussi remarquer que parmi tous les corps qu'il montre, et contrairement à ce que l'on a pu dire, le premier cinéma ne montre pas de phénomènes, de monstres (Delphin et Little Tich sont des artistes et non des monstres, et seul un Kobelkoff pourrait constituer une exception). Ces corps pathétiques et trop crûment présents, dont l'exhibition (sur l'échafaud ou dans les foires) était en sursis, ne pouvaient sans doute intégrer aisément les nouveaux modes de représentation.

C'est en somme le spectacle du corps réel, immédiat, qui est devenu tabou. Le fébrile questionnement fin de siècle sur le corps, la quête obsessionnelle de sa vérité, qui avaient suscité le foisonnement des spectacles des corps, se résolvent ou s'épuisent dans le cinéma, un cinéma qui peut montrer tous les corps, en viendra à le faire en effet, mais en les mettant à distance. C'est là que, tout d'ambiguïtés et de fauxsemblants, il prend sa place, après l'effervescence, dans l'effacement de spectacles devenus obsolètes.

Le paradoxe, c'est évidemment que ce nouveau régime de visibilité médiat, distancié, ait dû bientôt s'accommoder des visions atroces, sans fard et sans phrases, des corps déchirés de la Grande Guerre.

\section{NOTES}

1. Nous en citerons un certain nombre chemin faisant.

2. Dominique Kalifa, « Au rythme de la culture de masse », Paris 1900. La ville spectacle, Paris, Paris Musées, 2014, p. 27.

3. Émile Littré, Dictionnaire de la langue française, Paris, Hachette, 1874, t. IV, p. 2029.

4. Nous avons notamment délibérément laissé de côté les domaines bien connus des spectacles optiques et de la chronophotographie. 
5. Sur la danse en général, voir notamment: Anne Suquet, L'Éveil des modernités. Une histoire culturelle de la danse (1870-1945), Pantin, Centre national de la danse, 2012. Sur les relations de la danse, du rythme et du cinéma, voir Laurent Guido, L'Âge du rythme. Cinéma, musicalité et culture du corps dans les théories françaises des années 1910-1930, Payot, Lausanne, 2007

6. Paul Adam, «Critique des mœurs", Entretiens politiques et littéraires, n 36, 10 février 1893, p. 136.

7. Sur le café-concert, coir notamment : Concetta Condemi, Les Cafés-concerts, Paris, Quai Voltaire, 1992. Sur les relations entre le café-concert, le premier cinéma et les «mises en scène du corps pathologique ", voir Rae Beth Gordon, Why the French Love Jerry Lewis. From cabaret to early cinema, Stanford University Press, Stanford, California, 2001, traduit en français sous le titre : De Charcot à Charlot. Mises en scène du corps pathologique, Rennes, PUR, 2013.

8. Jeanne Bloch, la plus comique du monde entier, affiche de Daniel de Losques, 1908, reproduite dans : François Caradec et Alain Weill, Le Café-concert.1848-1914, Paris, Fayard, 2007, p. 266.

9. « Entre cour et jardin. Scala. Réouverture ", Journal amusant, n 430, 21 septembre 1907, p. 11.

10. Raitif de la Bretonne [pseudonyme de Jean Lorrain], "Pall Mall semaine », Le Journal, 6 décembre 1899, p. 1.

11. Sur prestidigitation et cinéma, voir Maxime Scheinfeigel, Cinéma et magie, Paris Armand Colin, 2008, et Frédéric Tabet, Le Cinématographe des magiciens. 1896-1906, un cycle magique, Rennes, PUR, 2018.

12. Sur la pantomime, voir notamment: Ariane Martinez, La Pantomime, théâtre en mineur. 1880-1945, Paris, Presses universitaires de la Sorbonne, 2008.

13. Charles Hacks, Le Geste, Paris, librairie Marpon et Flammarion, s.d. [1892].

14. Georges Polti, Notation des gestes, Paris, Albert Savine, s.d. [1892].

15. Charles Aubert, La Mimique, Paris, E. Meuriot, 1901.

16. Par exemple dans Le Petit Journal, dans Comœdia, dans Le XIX ${ }^{e}$ siècle, dans Le Journal.

17. Imposons la revue nue, revue représentée au concert des Folies royales en avril 1908.

18. Paris tout nu, revue représentée au concert des Ambassadeurs en mai 1908.

19. Nue, cocotte!, revue représentée au concert de la Cigale en juin 1908.

20. As-tu vu mon nu ?, revue représentée au concert des Ambassadeurs en août 1908.

21. J'veux du nu, na !, revue représentée au théâtre Montparnasse en octobre 1908.

22. À nu les femmes !, revue présentée au concert du Ba-Ta-Clan en décembre 1908.

23. Gustave-Joseph Witkowski et Lucien Nass, Le Nu au théâtre depuis l'Antiquité jusqu'à nos jours, Paris, Daragon, 1909, p. 1.

24. Ibid.

25. Jugement de la $9^{\mathrm{e}}$ chambre du tribunal de la Seine, Gazette des tribunaux, n. 25042, 27 et 28 juillet 1908, p. 670.

26. Yves Guyot, La Prostitution, Paris, G. Charpentier, 1882, p. 200.

27. Guyot-Daubès, Les hommes-phénomènes. Force, agilité, adresse, Paris, G. Masson, 1885.

28. Georges Strehly, L'Acrobatie et les acrobates, Paris, C. Delagrave, s.d. [1903], p. 39.

29. Ibid.

30. Edmond Desbonnet et Georges Rouhet, L'Art de créer le pur-sang humain, préfaces de Georges Strehly et Albert Surier, Paris, Nancy, Berger Levrault et cie, 1908.

31. Louis Guinard, Précis de tératologie: anomalies et monstruosités chez l'homme et chez les animaux, Paris, J. B. Baillière et fils, 1893.

32. Exposition de 1889. Guide bleu du Figaro et du Petit Journal, Paris, impr. de Chaix, 1889, p. 283.

33. Sur cinéma et sciences du psychisme, voir Mireille Berton, Le corps nerveux des spectateurs. Cinéma et sciences du psychisme autour de 1900, Lausanne, L’Âge d'Homme, 2015.

34. Affiche du musée de la Porte Saint-Denis, v. 1900, reproduite dans: Jacques Deslandes et Jacques Richard, Histoire comparée du cinéma, t. II, Du cinématographe au cinéma, 1896-1906, Bruxelles, Casterman, 1968, p. 15. 
35. Ibid.

36. Sur la morgue, v. Bruno Bertherat, La Morgue de Paris au XIX siècle (1804-1907). Les origines de l'institut médico-légal ou Les métamorphoses de la machine, thèse de doctorat, université de Paris 1 , 2003. Sur les relations de l'exposition à la morgue et l'émergence du cinéma, voir Vanessa Schwartz, Spectacular Realities: Early Mass Culture in fin-de-siècle Paris, Berkeley, University of California Press, 1999.

37. Ernest Cherbuliez, "La Morgue de Paris », La Revue des deux mondes, janvier-février 1891, p. 344-381, p. 344.

38. «Peugnez sous la guillotine », Paris, 2 février 1899, p. 2.

39. Octave Mirbeau, «Les joyeusetés de la peine de mort », Le Gaulois, 24 avril 1885, p. 1.

40. Documents sur Eusapia Palladino, Institut général psychologique. Section des recherches psychiques et physiologiques, Paris, au siège de la Société, 1909, p. 479.

41. Catalogue illustré. Musée Grévin, 1891, n.p.

42. Ibid.

43. Prospectus du Grand Musée Buiron, s.d. [v. 1901], collection Adrian, Bibliothèque nationale de France.

44. « Les maquettes animées », Le Monde illustré, 19 avril 1890, p. 250.

45. Notice du Théâtre mécanique de John Hewelt de Saint-Genois, s.l.n.d., n.p., Bibliothèque nationale de France.

46. Arthur Pougin, « Le théâtre et les spectacles à l'Exposition universelle de 1900 », Le Ménestrel, 13 janvier 1901, p. 12-13, p. 13.

47. Sur tableaux vivants et cinéma, voir Valentine Robert, L'Origine picturale du cinéma. Le tableau vivant, une esthétique du film des premiers temps, thèse de doctorat dirigée par François Albera, université de Lausanne, 2016.

48. Prospectus du Musée vivant Bonnefois, coll. Adrian, Bibliothèque nationale de France.

49. Henry Thétard, La Merveilleuse Histoire du cirque, Paris, Julliard, 1978, p. 11.

50. "Séance de la société générale des prisons du 20 décembre 1911 ", Revue pénitentiaire et de droit pénal, Paris, Marchal et Godde, janvier 1912, p. 41-86, p. 76.

51. Léonce Manouvrier, «Observations sur quelques nains ", Bulletins de la Société d'anthropologie de Paris, IV érie. t. VIII, 1897, p. 654-664.

52. Le comique Little Tich, qui mesurait $1,37 \mathrm{~m}$, avait six doigts à chaque main.

53. Cf. supra, note 7.

54. «Faits divers. La fête des loges ", La Patrie, 7 septembre 1880, p. 3.

55. « Le cerveau de Pranzini », Le Petit Journal, 4 septembre 1887, p. 2.

56. Adolphe Guillot, Paris qui souffre, la basse geôle du Grand-Châtelet et les morgues modernes, Paris, P. Rouquette, 1887.

57. «Fêtes foraines à Paris. Conditions de leur autorisation », Revue municipale, 11 juillet 1903, p. $428-430$, p. 429.

58. Ibid.

59. Voir Christiane Py et Cécile Vidart, «Les musées d'anatomie sur les champs de foire ", Actes de la recherche en sciences sociales, vol. 60, novembre 1985, p. 3-10, ici p. 9.

60. Sur les cours d'anatomie à l'école de Beaux-arts, voir Philippe Comar (dir.), Figures du corps, Paris, Beaux-arts de Paris les éditions, 2008.

61. Geoffrey Gorer, « Pornography of Death », Encounter, octobre 1955, p. 49-52. 


\section{RÉSUMÉS}

Les relations entre le cinéma et d'autres spectacles des corps, contemporains de son émergence, ont fait l'objet d'un certain nombre de travaux, parfois teintés de téléologie, souvent simplement continuistes, en tout cas limités à quelques spectacles. Cet article propose au contraire d'envisager l'ensemble des très nombreux spectacles de la fin du XIX ${ }^{\mathrm{e}}$ siècle dans lesquels le corps est le principal objet de l'attention (sans considération de possibles liens avec le cinéma), de discerner, dans cette vaste entreprise de monstration, des cohérences, de repérer des lignes d'évolution, et de voir, dès lors, quelle place y prend le cinéma.

The relationship between cinema and other spectacles of the body, contemporary with its emergence, has been the subject of a certain number of works, sometimes tinged with teleology, often simply continuist, but in any case limited to a few spectacles. This article proposes, on the contrary, to consider all of the numerous spectacles of the end of the 19th century in which the body is the main object of attention (without taking into consideration possible links to cinema), to discern, in this vast enterprise of monstration, coherences, to identify lines of evolution, and to see, thenceforth, what place cinema takes in it.

\section{INDEX}

Mots-clés : spectacles des corps, spectacles de la mort, spectacles de la nudité, premier cinéma, régime de visibilité

\section{AUTEUR}

\section{PATRICK DÉSILE}

THALIM UMR CNRS 